\title{
MONOMIAL PRINCIPALIZATION IN THE SINGULAR SETTING
}

\author{
COREY HARRIS
}

\begin{abstract}
We generalize an algorithm by Goward for principalization of monomial ideals in nonsingular varieties to work on any scheme of finite type over a field. The normal crossings condition considered by Goward is weakened to the condition that components of the generating divisors meet as complete intersections. This leads to a substantial generalization of the notion of monomial scheme; we call the resulting schemes 'regular crossings (r.c.) monomial.' We prove that r.c. monomial subschemes in arbitrarily singular varieties can be principalized by a sequence of blow-ups at codimension 2 r.c. monomial centers.
\end{abstract}

1. Introduction. Monomial schemes are schemes defined as intersections of collections of components from a fixed normal crossing divisor in a nonsingular variety. In [4], Goward proves that monomial schemes may be principalized by a sequence of blow-ups along codimension 2 monomial schemes. In the singular setting, this definition of monomial scheme is not available, because the notion of normal crossings requires a nonsingular ambient variety. We consider a much weaker condition, which makes no nonsingularity assumption on the ambient variety: essentially, divisors meet with 'regular crossings' if they intersect along subschemes with the expected dimension. (For example, any two distinct irreducible curves on a smooth surface meet with regular crossings, regardless of whether they are nonsingular or meet transversally.) This leads to a generalization of the notion of monomial scheme, 'r.c. monomial schemes.' See Section 2 for formal definitions.

We extend Goward's result to the r.c. monomial case, showing that this much larger class of subschemes can be principalized via Goward's procedure.

Our result has been used in recent work on computations of Segre classes, cf., [1, Theorem 1.1].

2010 AMS Mathematics subject classification. Primary 14B05.

Received by the editors on April 4, 2014. 
2. Definitions and examples. Throughout, $X$ will denote a scheme of finite type over an arbitrary field. By regular sequence, we mean a sequence $x_{1}, \ldots, x_{n}$ of elements in a ring $R$ such that $\left(x_{1}, \ldots, x_{n}\right) \subset R$ is a proper ideal and, for each $i$, the image of $x_{i}$ in $R /\left(x_{1}, \ldots, x_{i-1}\right)$ is a non-zerodivisor, see [2, page 243].

Definition (Regular crossings). Let $Y_{1}, \ldots, Y_{n} \subset X$ be Cartier divisors. We say that $\left\{Y_{1}, \ldots, Y_{n}\right\}$ has regular crossings if, for every subset $A \subset\left\{Y_{1}, \ldots, Y_{n}\right\}$ and every point $p \in \cap_{Y \in A} Y$, the local equations $y_{i}$ for the $Y \in A$ form a regular sequence at $p$.

Note that the definition requires each $Y_{i}$ to be cut out locally by a non-zerodivisor, making $Y_{i}$ an effective Cartier divisor in $X$. Note also that the condition places no restrictions on $X$.

The following definition will be used only in the introduction to compare concepts.

Definition (Simple normal crossings). Let $Y_{1}, \ldots, Y_{n} \subset X$ be Cartier divisors. We say that $\left\{Y_{1}, \ldots, Y_{n}\right\}$ has simple normal crossings if, for every subset $A \subset\left\{Y_{1}, \ldots, Y_{n}\right\}$, the intersection $Z=\cap_{Y \in A} Y$ is nonsingular with $\operatorname{codim}_{X} Z=\# A$. If $D=\sum a_{i} Y_{i}$, with $a_{i} \geq 0$, we say $D$ is a simple normal crossings divisor or s.n.c divisor.

The s.n.c. condition on singletons requires each $Y_{i}$ to be nonsingular, and the condition on the empty set means $X$ itself must be nonsingular.

Example 2.1. Let $\mathbb{A}_{k}^{2}=\operatorname{Spec} k[x, y]$ and consider the curves $Y_{1}$ defined by $y$ and $Y_{2}$ defined by $y^{2}-x^{3}$. These two curves do not meet with simple normal crossings because $y^{2}-x^{3}$ is not smooth in the intersection. So $Y_{1}+Y_{2}$ is not an s.n.c. divisor, but $\left\{Y_{1}, Y_{2}\right\}$ does have regular crossings since $y^{2}-x^{3}$ is sent to a non-zerodivisor in the integral domain $k[x, y] /(y)$. Observe (Figure $1(\mathrm{~A})$ ) that these curves do not meet transversally.

Example 2.2. Now consider the cone $C=\operatorname{Spec} k[x, y, z] /\left(x y-z^{2}\right)$ and the subschemes $Y_{x}, Y_{y}$ cut out by the ideals $(x)$ and $(y)$, respectively. Since $C$ is singular, there are no s.n.c. divisors in sight. However, 


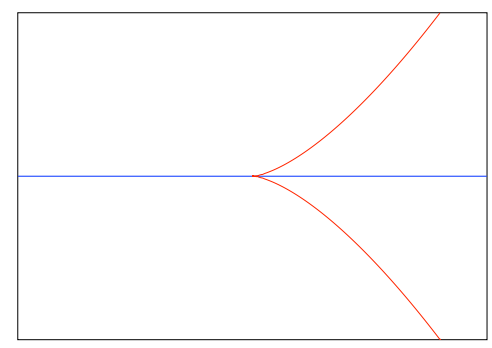

(A) Curves $y, y^{2}-x^{3}$ in the plane

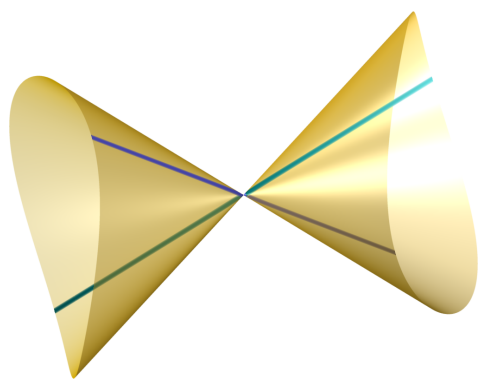

(B) Curves $x, y$ on $x y-z^{2}$

Figure 1. Examples.

$\left(k[x, y, z] /\left(x y-z^{2}\right)\right) /(y) \cong k[x, z] /\left(z^{2}\right)$, so $y, x$ forms a regular sequence at every point in $C$. Thus, $\left\{Y_{x}, Y_{y}\right\}$ has regular crossings in $C$.

Remark 2.3. In both of the above examples, we checked the r.c. crossing condition only affine-locally, even though the definition is in terms of stalks. This is sufficient because if $x_{1}, \ldots, x_{n}$ is a regular sequence in a Noetherian ring $R$, then it remains a regular sequence in $R_{p}$ for any prime ideal $p \subset R$ containing $\left\{x_{1}, \ldots, x_{n}\right\}$.

Definition. A subscheme $Z \subset X$ is called monomial if it is cut out by effective divisors which are supported on a fixed s.n.c. divisor. By analogy, if $\left\{Y_{1}, \ldots, Y_{n}\right\}$ has regular crossings, we will say $Z$ is an r.c. monomial subscheme (with respect to $\left\{Y_{1}, \ldots, Y_{n}\right\}$ ) if $Z$ is cut out by divisors of the form $\sum a_{i} Y_{i}$ with $a_{i} \geq 0$. As well, if $\beta: \widetilde{X} \rightarrow X$ is the blowup of $X$ at an r.c. monomial subscheme, we will call $\beta$ (or just $\widetilde{X}$ ) an r.c. monomial blowup (with respect to $\left\{Y_{1}, \ldots, Y_{n}\right\}$ ).

Theorem 2.4 (Main theorem). Let $\left\{Y_{1}, \ldots, Y_{n}\right\}$ have regular crossings on $X$. If $D_{1}, \ldots, D_{h}$ are given by $D_{j}=\sum a_{i j} Y_{i}$, where $a_{i j}>0$, then there exists a sequence of r.c. monomial blowups at codimension 2 centers

$$
\tilde{X}=X_{n} \stackrel{\beta_{n}}{\longrightarrow} X_{n-1} \stackrel{\beta_{n-1}}{\longrightarrow} \cdots \stackrel{\beta_{2}}{\longrightarrow} X_{1} \stackrel{\beta_{1}}{\longrightarrow} X
$$

such that $\left(\mathscr{I}_{D_{1}}+\cdots+\mathscr{I}_{D_{h}}\right) \mathcal{O}_{X_{i}}$ is r.c. monomial for each $i$ and $\left(\mathscr{I}_{D_{1}}+\cdots+\mathscr{I}_{D_{h}}\right) \mathcal{O}_{\widetilde{X}}$ is locally principal. 
Goward's theorem [4, Theorem 2] is the analogous statement to Theorem 2.4 for monomial subschemes. The algorithm here is a direct generalization of Goward's, and the proof in Section 4 is essentially a summary of [4, Sections 2-3]. We first need to know that r.c. monomial blowups preserve regular crossings, and so we verify this in Section 3.

3. R.c. monomial blowups preserve regular crossings. Assume $\left\{Y_{1}, \ldots, Y_{n}\right\}$ has regular crossings and $n \geq 2$. Let $D_{1}=\sum a_{i} Y_{i}$ and $D_{2}=\sum b_{i} Y_{i}$ with $a_{i}, b_{j} \geq 0$. If $\beta: \widetilde{X} \rightarrow X$ be the blowup of $X$ at $Y_{1} \cap Y_{2}$, let $E$ denote the exceptional divisor in $\widetilde{X}$, and let $\widetilde{Y}_{i}$ denote the proper transform of $Y_{i}$ in $\widetilde{X}$. We will verify that $\beta^{*} D_{i}$, which is supported on $\left\{E, \widetilde{Y}_{1}, \ldots, \widetilde{Y}_{n}\right\}$, has regular crossings.

The proof will be by induction on the number of divisors. The base case is handled in Proposition 3.1, and the inductive step is handled in Proposition 3.3.

Proposition 3.1. Suppose $\left\{Y_{1}, Y_{2}\right\}$ has regular crossings on $X$. Let $\beta: \widetilde{X} \rightarrow X$ denote the blowup of $X$ at $Y_{1} \cap Y_{2}$. Then $\left\{E, \widetilde{Y}_{1}, \widetilde{Y}_{2}\right\}$ has regular crossings in $\tilde{X}$.

Proof. There are eight subsets of $\left\{\widetilde{Y_{1}}, \widetilde{Y_{2}}, E\right\}$. The intersections corresponding to $\emptyset,\left\{\widetilde{Y_{1}}, \widetilde{Y_{2}}\right\},\left\{\widetilde{Y_{1}}, \widetilde{Y_{2}}, E\right\}$ are empty, and those corresponding to $\left\{\widetilde{Y_{1}}\right\},\left\{\widetilde{Y_{2}}\right\},\{E\}$ are effective Cartier. This leaves the intersections corresponding to $\left\{\widetilde{Y_{1}}, E\right\}$ and $\left\{\widetilde{Y_{2}}, E\right\}$. We show the result for $\left\{\widetilde{Y_{1}}, E\right\}$.

It suffices to prove the result affine-locally. Since $Y_{1}$ and $Y_{2}$ are effective Cartier divisors on $X$, there is an affine open cover $\left\{U_{\alpha}=\right.$ Spec $\left.R_{\alpha}\right\}$ of $X$ such that $y_{1, \alpha}$ is a local equation for $Y_{1}$ on $U_{\alpha}$ and $y_{2, \alpha}$ is a local equation for $Y_{2}$. Now let $U=\operatorname{Spec} R$ be a member of such a cover, and let $\left(y_{1}\right),\left(y_{2}\right) \subset R$ be principal ideals defining $Y_{1}$ and $Y_{2}$, respectively. Then the blowup $\widetilde{U}$ of $U$ centered at $Y_{1} \cap Y_{2}$ is $\operatorname{Proj} R\left[a_{1}, a_{2}\right] /\left(a_{2} y_{1}-a_{1} y_{2}\right)$, see [3, B.6.10]. Consider the open set $D\left(a_{2}\right):=\left\{[p] \in \operatorname{Spec} R \mid a_{2} \notin p\right\}$ which we can write as

$$
D\left(a_{2}\right)=\operatorname{Spec} R\left[a_{1}\right] /\left(y_{1}-a_{1} y_{2}\right) .
$$

The pullback $\beta^{*} Y_{1}$ is cut out by $a_{1} y_{2}$ in $D\left(a_{2}\right)$ and the exceptional divisor is cut out by $y_{2}$, so the proper transform of $Y_{1}$ is cut out in 
$D\left(a_{2}\right)$ by $a_{1}$. Since $\left(R\left[a_{1}\right] /\left(y_{1}-a_{1} y_{2}\right)\right) /\left(a_{1}\right) \cong R /\left(y_{1}\right)$, and $y_{2}$ is not a zerodivisor in $R /\left(y_{1}\right)$ by assumption, we have that $a_{1}, y_{2}$ is a regular sequence in $R\left[a_{1}\right] /\left(y_{1}-a_{1} y_{2}\right)$ corresponding to the intersection $Y_{1} \cap Y_{2}$. The argument for $D\left(a_{1}\right)$ is analogous.

The proof is completed by noting again (Remark 2.3) that localization preserves regular sequences, so that $a_{1}, y_{2}$ is a regular sequence in $\mathcal{O}_{\widetilde{X}, p}$ for each $p \in \widetilde{Y}_{1} \cap E$.

We note that as a scheme of finite type over a Noetherian ring, $X$ is Noetherian. In particular, $\mathcal{O}_{X, p}$ is a Noetherian local ring for all $p \in X$. In the proof of Proposition 3.3, we will need the following result.

Lemma 3.2. If $R$ is a Noetherian local ring and $x_{1}, \ldots, x_{r}$ is a regular sequence of elements in the maximal ideal of $R$, then any permutation of $x_{1}, \ldots, x_{r}$ is again a regular sequence.

Proof. See [2, Theorem 17.2].

We will make repeated use of the lemma, along with the idea that if $x_{1}, \ldots, x_{n}$ is a regular sequence, then $x_{1}, \ldots, x_{k}$ is a regular sequence for $1 \leq k \leq n$.

Proposition 3.3. Suppose $\left\{Y_{1}, \ldots, Y_{n}\right\}$ has regular crossings on $X$. Let $\beta: \widetilde{X} \rightarrow X$ denote the blowup of $X$ at $Y_{1} \cap Y_{2}$. Then $\left\{E, \widetilde{Y}_{1}, \ldots, \widetilde{Y}_{n}\right\}$ has regular crossings in $\widetilde{X}$.

Proof. By induction, assume $\left\{E, \widetilde{Y}_{1}, \ldots, \widetilde{Y}_{n-1}\right\}$ has regular crossings. We will show that if $A$ contains any (possibly empty) subset of $\left\{E, \widetilde{Y}_{1}, \widetilde{Y}_{2}\right\}$, then the corresponding intersection is cut out by a regular sequence at each point in an affine chart on the blowup.

Let $A \subset\left\{E, \tilde{Y}_{1}, \ldots, \tilde{Y}_{n}\right\}$. We want $\cap_{Z \in A} Z \neq \emptyset$ so $\left\{\tilde{Y}_{1}, \tilde{Y}_{2}\right\}$ cannot be a subset of $A$. Assume then without loss of generality that $\tilde{Y}_{2} \notin A$. Assume also that $\widetilde{Y}_{n} \in A$. Each element of $\left\{Y_{1}, \ldots, Y_{n}\right\}$ is an effective Cartier divisor so again we can find a cover of $X$ by open affines $\left\{U_{\alpha}=\operatorname{Spec} R_{\alpha}\right\}$ such that for each $i$, and each $Y \in\left\{Y_{1}, \ldots, Y_{n}\right\}$, we have a non-zerodivisor $y \in R_{\alpha}$ which cuts out $Y$ in $U_{\alpha}$. Choose such a $U=\operatorname{Spec} R$, and let $y_{i} \in R$ be a local equation for $Y_{i}$. We can 
then write the blowup of this chart $\widetilde{U}=\operatorname{Proj} R\left[a_{1}, a_{2}\right] /\left(a_{2} y_{1}-a_{1} y_{2}\right)$. As before, we work affine-locally in $D\left(a_{2}\right)=\operatorname{Spec} R\left[a_{1}\right] /\left(y_{1}-a_{1} y_{2}\right)$, and we have that $E$ is cut out by $y_{2}$ and $\widetilde{Y}_{1}$ is cut out by $a_{1}$.

Assume that $A$ contains both $E$ and $\widetilde{Y}_{1}$. By induction we can find a regular sequence for $A \backslash\left\{\widetilde{Y}_{n}\right\}$ in the elements $\left\{y_{2}, a_{1}, s_{1}, \ldots, s_{k}\right\}$ (where $\left.\#\left(A \backslash\left\{\tilde{Y}_{n}\right\}\right)=k+2\right)$ and so Lemma 3.2 ensures that $a_{1}, y_{2}, s_{1}, \ldots, s_{k}$ is a regular sequence at each $p \in \cap_{Y \in A \backslash\left\{\widetilde{Y}_{n}\right\}} Y$. Let $r$ be a local equation for $\tilde{Y}_{n}$ on $D\left(a_{2}\right)$ such that we have a regular sequence in the elements $r, y_{1}, y_{2}, s_{1}, \ldots, s_{k}$ at each point $p \in \cap_{Y \in A} Y$. Then, by Lemma 3.2, these elements form a regular sequence in any order. Thus, $y_{2}, r, s_{1}, \ldots, s_{n}$ form a regular sequence in $R /\left(t_{1}\right)$, showing that $a_{1}, y_{2}, r, s_{1}, \ldots, s_{n}$ is a regular sequence at each point $p \in \cap_{Y \in A} Y$.

If $A$ contains $E$ but does not contain $\widetilde{Y}_{1}$, then we can use Lemma 3.2 again to get the regular sequence $s_{1}, \ldots, s_{n}, r, y_{2}, a_{1}$ which shows that $s_{1}, \ldots, s_{n}, r, y_{2}$ is a regular sequence. Similarly, if $A$ contains $\widetilde{Y}_{1}$ but not $E$, we can rearrange to get $s_{1}, \ldots, s_{n}, r, a_{1}, y_{2}$, which shows $s_{1}, \ldots, s_{n}, r, a_{1}$ is a regular sequence. Truncating this sequence again shows that $s_{1}, \ldots, s_{n}, r$ is a regular sequence. This is the case where $A$ contains neither $E$ nor $\widetilde{Y}_{1}$.

In this paper, we will only need the result as stated in Proposition 3.3, but since the fact is true in greater generality, we provide Proposition 3.4 for completeness. In the following proof, we use Proposition 3.3 as the base case and induct on the number of divisors that cut out the center for the blowup.

Proposition 3.4. Suppose $\left\{Y_{1}, \ldots, Y_{n}\right\}$ has regular crossings on $X$. Let $\beta: \widetilde{X} \rightarrow X$ denote the blowup of $X$ at $\cap_{i=1}^{r} Y_{i}$, where $r \leq n$. Then $\left\{E, \widetilde{Y}_{1}, \ldots, \widetilde{Y}_{n}\right\}$ has regular crossings in $\widetilde{X}$.

Proof. Let $A \subset\left\{E, \widetilde{Y}_{1}, \ldots, \widetilde{Y}_{n}\right\}$ with $\widetilde{Y}_{1} \in A$. We want $\cap_{Z \in A} Z \neq \emptyset$, so we require $A \cap\left\{\widetilde{Y}_{2}, \ldots, \widetilde{Y}_{r}\right\}=\emptyset$. Each element of $\left\{Y_{1}, \ldots, Y_{n}\right\}$ is an effective Cartier divisor, so again we can find a cover of $X$ by open affines $\left\{U_{\alpha}=\operatorname{Spec} R_{\alpha}\right\}$ such that, for each $\alpha$, and each $Y \in\left\{Y_{1}, \ldots, Y_{n}\right\}$, we have a non-zerodivisor $y \in R_{\alpha}$ which cuts out $Y$ in $U_{\alpha}$. Choose such a $U=\operatorname{Spec} R$, and let $y_{i} \in R$ be a local equation 
for $Y_{i}$. We can then write the blowup of this chart

$$
\widetilde{U}=\operatorname{Proj} R\left[a_{1}, \ldots, a_{r}\right] /\left(\left\{a_{j} y_{i}-a_{i} y_{j} \mid i \neq j\right\}\right)
$$

(again see [3, B.6.10]). As before, we work affine-locally in

$$
D\left(a_{1}\right)=\operatorname{Spec} R\left[a_{2}, \ldots, a_{r}\right] /\left(\left\{y_{i}-a_{i} y_{1} \mid i \neq 1\right\}\right)
$$

where we have that $E$ is cut out by $y_{1}$, and $\widetilde{Y}_{i}$ is cut out by $a_{i}$ for each $i>2$.

We can use induction on $r$ with the base case Proposition 3.3. Then assume $\left\{E, \widetilde{Y}_{1}, \ldots, \widetilde{Y}_{n}\right\}$ has regular crossings in the blowup $\beta^{\prime}: X^{\prime} \rightarrow X$ centered at $\cap_{i=1}^{r-1} Y_{i}$. If we let $U^{\prime}$ denote the blowup of the chart $U$ by $\beta^{\prime}$, then

$$
U^{\prime}=\operatorname{Proj} R\left[a_{1}, \ldots, a_{r-1}\right] /\left(\left\{a_{j} y_{i}-a_{i} y_{j} \mid i \neq j\right\}\right)
$$

with corresponding open chart

$$
D^{\prime}\left(a_{1}\right)=\operatorname{Spec} R\left[a_{1}, \ldots, a_{r-1}\right] /\left(\left\{y_{i}-a_{i} y_{1} \mid i=2, \ldots, r-1\right\}\right) .
$$

Now we observe that $D\left(a_{1}\right)$ is related to $D^{\prime}\left(a_{1}\right)$ by

$$
\begin{aligned}
D\left(a_{1}\right) \cap \mathbb{V}\left(a_{r}\right) & =\operatorname{Spec}\left(R\left[a_{2}, \ldots, a_{r}\right] /\left(\left\{y_{i}-a_{i} y_{1} \mid i \neq 1\right\}\right)\right) /\left(a_{r}\right) \\
& =\operatorname{Spec} R\left[a_{2}, \ldots, a_{r-1}\right] /\left(\left\{y_{i}-a_{i} y_{1} \mid i \neq 1, r\right\} \cup\left\{y_{r}\right\}\right) \\
& =D^{\prime}\left(a_{1}\right) \cap \mathbb{V}\left(y_{r}\right) .
\end{aligned}
$$

Recall that $\widetilde{Y}_{r} \notin A$. The inductive hypothesis says that we can find a regular sequence $s_{1}, \ldots, s_{k}$ corresponding to $A$, where $k=\# A$. Then, as usual, we can localize at a point and rearrange to get that $y_{r}, s_{1}, \ldots, s_{k}$ is a regular sequence at each point $p \in D^{\prime}\left(a_{1}\right)$. Then $s_{1}, \ldots, s_{k}$ is a regular sequence at each point in $D^{\prime}\left(a_{1}\right) /\left(y_{r}\right)=$ $D\left(a_{1}\right) /\left(a_{r}\right)$, so $a_{r}, s_{1}, \ldots, s_{k}$ is a regular sequence at each point of $D\left(a_{1}\right)$.

4. Proof of main theorem. In [4], Goward defines an invariant $(\sigma, \tau)$ on the divisors in question. We adopt those definitions for the new context here: 
Definition. Let $D_{1}=\sum_{i=1}^{n} a_{i} Y_{i}$ and $D_{2}=\sum_{i=1}^{n} b_{i} Y_{i}$ where $\left\{Y_{1}, \ldots\right.$, $\left.Y_{n}\right\}$ has regular crossings in $X$ and $a_{i}, b_{j} \geq 0$. We define

$$
\sigma_{i j}\left(D_{1}, D_{2}\right)=\max \left\{\left(\left|a_{i}-b_{i}\right|,\left|a_{j}-b_{j}\right|\right),\left(\left|a_{j}-b_{j}\right|,\left|a_{i}-b_{i}\right|\right)\right\}
$$

if $a_{i}-b_{i}$ and $a_{j}-b_{j}$ have opposite signs, and otherwise

$$
\sigma_{i j}\left(D_{1}, D_{2}\right)=(-\infty,-\infty),
$$

where the max is taken lexicographically. Now we can define

$$
\begin{aligned}
& \sigma\left(D_{1}, D_{2}\right)=\max \left\{\sigma_{i j}\left(D_{1}, D_{2}\right) \mid Y_{i} \cap Y_{j} \neq 0, \quad i \neq j\right\} \\
& \tau\left(D_{1}, D_{2}\right)=\#\left\{(i, j) \mid \sigma_{i j}\left(D_{1}, D_{2}\right)=\sigma\left(D_{1}, D_{2}\right), \quad i \leq j\right\},
\end{aligned}
$$

so $\sigma\left(D_{1}, D_{2}\right)$ takes the value of the worst intersection in the support, and $\tau\left(D_{1}, D_{2}\right)$ counts how many intersections share this value.

These invariants are calculated for divisors on $X$ and, after blowing up, for their pullbacks. We show that these calculations go the same way as in the simple normal crossings context and then outline the steps of the proof.

Proposition 4.1. Let $D_{1}$ and $D_{2}$ be as defined above. Then $\mathscr{I}_{D_{1}}+\mathscr{I}_{D_{2}}$ is principal at $p \in X$ if and only if $\sigma_{i j}\left(D_{1}, D_{2}\right)=(-\infty,-\infty)$ whenever $p \in Y_{i} \cap Y_{j}$.

Proof. Let $x_{1}, \ldots, x_{n}$ be a regular sequence at $p \in X$ corresponding to $Y_{1}, \ldots, Y_{n}$. Then, let $f_{1}=u_{1} x_{1}^{a_{1}} \cdots x_{n}^{a_{n}}$ and $f_{2}=u_{2} x_{1}^{b_{1}} \cdots x_{n}^{b_{n}}$ be local equations for $D_{1}$ and $D_{2}$, where $u_{i} \in \mathcal{O}_{X, p}$ are units. Suppose $\mathscr{I}_{D_{1}}+\mathscr{I}_{D_{2}}$ is not principal at $p$. Then $\left(f_{1}, f_{2}\right)$ is not principal, so we have some $i, j$ such that $a_{i}-b_{i}$ and $a_{j}-b_{j}$ have opposite signs. Thus, $\sigma_{i j}\left(D_{1}, D_{2}\right)>0$. On the other hand, suppose $p \in Y_{i} \cap Y_{j}$ and $\sigma_{i j}\left(D_{1}, D_{2}\right)>0$. Then $a_{i}-b_{i}$ and $a_{j}-b_{j}$ have opposite signs, so $\left(f_{1}, f_{2}\right)$ is not principal and thus $\mathscr{I}_{D_{1}}+\mathscr{I}_{D_{2}}$ is not principal at $p$.

As a result of this proof, we see that if $\mathscr{I}_{D_{1}}+\mathscr{I}_{D_{2}}$ is principal at $p$, and $\left(\mathscr{I}_{D_{1}}+\mathscr{I}_{D_{2}}\right)_{p}=\left(\mathscr{I}_{D_{i}}\right)_{p}$ for some $i \in\{1,2\}$. So by induction, we have that if $\mathscr{I}_{D_{1}}+\cdots+\mathscr{I}_{D_{h}}$ is principal at $p$, then $\left(\mathscr{I}_{D_{1}}+\cdots+\mathscr{I}_{D_{h}}\right)_{p}=\left(\mathscr{I}_{D_{i}}\right)_{p}$ for some $i \in\{1, \ldots, h\}$. 
We have left to show that blowing up at the chosen codimension 2 centers strictly reduces the invariant $(\sigma, \tau)$ and then that such blowups can be taken successively until $(\sigma, \tau)=(-\infty,-\infty)$.

Proposition 4.2. Let $D_{1}$ and $D_{2}$ be as defined above. Suppose we have $(i, j)$ such that $\sigma_{i j}\left(D_{1}, D_{2}\right)=\sigma\left(D_{1}, D_{2}\right)>(-\infty,-\infty)$, and let $\beta: \widetilde{X} \rightarrow X$ be the blowup of $X$ centered at $Y_{i} \cap Y_{j}$. Then

$$
\left(\sigma\left(D_{1}, D_{2}\right), \tau\left(D_{1}, D_{2}\right)\right)>\left(\sigma\left(\beta^{*} D_{1}, \beta^{*} D_{2}\right), \tau\left(\beta^{*} D_{1}, \beta^{*} D_{2}\right) .\right.
$$

Sketch of proof. Assume that $(i, j)=(1,2)$ so that the blowup is centered at $Y_{1} \cap Y_{2}$. The proof relies on calculations of $\sigma_{i j}\left(\beta^{*} D_{1}, \beta^{*} D_{2}\right)$, which depend only on the coefficients $a_{i}, b_{i}, a_{j}, b_{j}$. The details can be found in the proof of [4, Theorem 1]. Here we verify only that the calculations of $\sigma_{i j}\left(\beta^{*} D_{1}, \beta^{*} D_{2}\right)$ from the s.n.c. case still go through with regular crossings.

Let $E \subset \widetilde{X}$ denote the exceptional divisor. Then

$$
\beta^{*} Y_{i}= \begin{cases}\tilde{Y}_{i}+E & \text { if } i=1,2 \\ \widetilde{Y}_{i} & \text { if } i>2\end{cases}
$$

as seen from the work done in Proposition 3.1. Thus,

$$
\beta^{*} D_{1}=\left(a_{1}+a_{2}\right) E+\sum_{i} a_{i} \widetilde{Y}_{i}
$$

and

$$
\beta^{*} D_{2}=\left(b_{1}+b_{2}\right) E+\sum_{i} b_{i} \tilde{Y}_{i}
$$

Theorem 2.4 (Main theorem). Let $\left\{Y_{1}, \ldots, Y_{n}\right\}$ have regular crossings on $X$. If $D_{1}, \ldots, D_{h}$ are given by $D_{j}=\sum a_{i j} Y_{i}$, where $a_{i j}>0$, then there exists a sequence of r.c. monomial blowups at codimension 2 centers

$$
\tilde{X}=X_{n} \stackrel{\beta_{n}}{\longrightarrow} X_{n-1} \stackrel{\beta_{n-1}}{\longrightarrow} \cdots \stackrel{\beta_{2}}{\longrightarrow} X_{1} \stackrel{\beta_{1}}{\longrightarrow} X
$$

such that $\left(\mathscr{I}_{D_{1}}+\cdots+\mathscr{I}_{D_{h}}\right) \mathcal{O}_{X_{i}}$ is r.c. monomial for each $i$ and $\left(\mathscr{I}_{D_{1}}+\cdots+\mathscr{I}_{D_{h}}\right) \mathcal{O}_{\widetilde{X}}$ is locally principal. 
Proof. By the previous remarks, we can assume $h=2$. Let $Y_{1}, \ldots, Y_{n}$ be the divisors in the support of $D_{1}+D_{2}$. If $\sigma\left(D_{1}, D_{2}\right)=$ $(-\infty,-\infty)$ we are done, so assume not. Then let

$$
(i, j)=\max \left\{(k, l) \mid \sigma_{k l}\left(D_{1}, D_{2}\right)=\sigma\left(D_{1}, D_{2}\right)\right\}
$$

where the $\max$ is taken lexicographically. If we take the blowup $\beta_{1}: X_{1} \rightarrow X$ centered at $Y_{i} \cap Y_{j}$, then Proposition 4.2 gives that

$$
\left(\sigma\left(D_{1}, D_{2}\right), \tau\left(D_{1}, D_{2}\right)\right)>\left(\sigma\left(\beta^{*} D_{1}, \beta^{*} D_{2}\right), \tau\left(\beta^{*} D_{1}, \beta^{*} D_{2}\right) .\right.
$$

Now we note that $\beta_{1}^{*} D_{1}$ and $\beta_{1}^{*} D_{2}$ are divisors supported on $\left\{E, \widetilde{Y}_{1}, \ldots\right.$, $\left.\widetilde{Y}_{n}\right\}$ and have regular crossings by Proposition 3.3. Thus, $\left(\mathscr{I}_{D_{1}}+\right.$ $\left.\mathscr{I}_{D_{2}}\right) \mathcal{O}_{\widetilde{X}}$ defines an r.c. monomial subscheme of $\widetilde{X}$.

We can repeat this process, with $(\sigma, \tau)$ decreasing at each iteration. Since $(\sigma, \tau)$ takes values in $\mathbb{N}^{2} \times \mathbb{N}$, we must get $(\sigma, \tau)=(-\infty,-\infty)$ after finitely many steps, yielding the desired sequence of blowups.

Acknowledgments. Thanks to Paolo Aluffi for suggesting this problem and for his patience and feedback while this work was prepared.

\section{REFERENCES}

1. Paolo Aluffi, Segre classes as integrals over polytopes, J. Eur. Math. Soc., to appear.

2. David Eisenbud, Commutative algebra: With a view toward algebraic geometry, Grad. Texts Math., Springer-Verlag, New York, 1995.

3. William Fulton, Intersection theory, 2nd edition, Ergeb. Math. Grenz. 2, Springer-Verlag, Berlin, 1998.

4. Russell A. Goward, Jr., A simple algorithm for principalization of monomial ideals, Trans. Amer. Math. Soc. 357 (2005), 4805-4812 (electronic).

Department of Mathematics, Florida State University, Tallahassee FL, 32306

Email address: charris@math.fsu.edu 